\title{
Epithelial Cell Adhesion Molecule
}

\section{More than a Carcinoma Marker and Adhesion Molecule}

\author{
Monika Trzpis, Pamela M.J. McLaughlin, \\ Lou M.F.H. de Leij, and Martin C. Harmsen \\ From the Department of Pathology and Laboratory Medicine, \\ University Medical Center Groningen, University of Groningen, \\ Groningen, The Netherlands
}

The epithelial cell adhesion molecule (ЕрCAM, CD326) is a glycoprotein of $\sim 40 \mathrm{kd}$ that was originally identified as a marker for carcinoma, attributable to its high expression on rapidly proliferating tumors of epithelial origin. Normal epithelia express EpCAM at a variable but generally lower level than carcinoma cells. In early studies, EPCAM was proposed to be a cell-cell adhesion molecule. However, recent insights revealed a more versatile role for $\mathrm{EPCAM}$ that is not limited only to cell adhesion but includes diverse processes such as signaling, cell migration, proliferation, and differentiation. Cell surface expression of EpCAM may actually prevent cell-cell adhesion. Here, we provide a comprehensive review of the current knowledge on ЕрСАM biology in relation to other cell adhesion molecules. We discuss the implications of the newly identified functions of ЕPCAM in view of its prognostic relevance in carcinoma, inflammatory pathophysiology, and tissue development and regeneration as well as its role in normal epithelial homeostasis. (Am J Pathol 2007, 171:386-395; DOI: 10.2353/ajpath.2007.070152)

Cell adhesion is a fundamental process required for the correct functioning of multicellular organisms. Many cell adhesion molecules (CAMs) have been characterized in recent decades and their function in different processes has been the subject of intense study. It has become clear that CAMs are involved in a broad range of processes, including cell-cell and cell-matrix interactions, cell migration, cell cycle, and signaling as well as morphogenesis during development and tissue regeneration. ${ }^{1}$ The pivotal role of CAMs is highlighted by the fact that CAMs are involved in a variety of pathologies ranging from cancer, inflammation, pathogenic infections to autoimmune disease. ${ }^{1}$

Four archetypal CAM families have been identified: the cadherins, the selectins, the integrins, and the immunoglobulin CAM (Ig-CAM) superfamily. Members of the cadherin family are calcium-dependent glycoproteins that contain an extracellular domain CAM with three to five internal repeats, a single-spanning transmembrane domain, and an intracellular domain. ${ }^{1}$ In selectins, the extracellular domain consists of a calcium-dependent lectin domain, an epidermal growth factor-like domain, followed by a domain homologous to epidermal growth factor, and two to nine consensus repeats. Furthermore, selectins contain a hydrophobic transmembrane domain and a short cytoplasmic tail. ${ }^{2}$ Integrins are composed of two noncovalently associated membrane-spanning subunits, designated $\alpha$ and $\beta$. The particular combination of $\alpha$ and $\beta$ subunits determines the specificity for the extracellular ligands and, thereby, determines the concomitant intracellular signaling events. ${ }^{3}$ The integrin family represents the major receptor family for interaction with the extracellular matrix. Members of the Ig-CAM family are calcium-independent CAMs, of which the extracellular domain is composed of a ligand-binding domain of four to six Ig-like repeats, up to five fibronectin-like repeats, a transmembrane domain, and an intracellular domain. $^{1}$

Additionally, several CAMs exist that do not share any of the structural patterns of the four CAM families. The most prominent example of this is the epithelial cell adhesion molecule (EpCAM). EpCAM was independently identified by many research groups, resulting in a plethora of synonyms (Table 1), although recently a unified nomenclature has been proposed with the terms "EpCAM" and CD326. ${ }^{4}$ The extracellular domain of EpCAM starts with a signal sequence that is followed by an epidermal growth factor-like repeat, a human thyroglobulin repeat (TY), and a cysteine-poor domain. ${ }^{4}$ EpCAM has a

Accepted for publication May 1, 2007.

Address reprint requests to Dr. M.C. Harmsen, UMCG, Department of Pathology and Medical Laboratory, Hanzeplein 1, 9713 GZ Groningen, The Netherlands. E-mail: m.c.harmsen@med.umcg.nl. 
Table 1. Names Proposed by Different Groups for EpCAM Are Associated with Monoclonal Antibodies Specific for the Cell Surface Antigen or cDNA Clones Used to Characterize the Antigen

\begin{tabular}{|c|c|}
\hline Abbreviation & Name \\
\hline \multicolumn{2}{|c|}{$\begin{array}{l}\text { Based on a monoclonal antibody specific for the cell surface } \\
\text { antigen }\end{array}$} \\
\hline CD326 & Cluster of differentiation $326^{64}$ \\
\hline GA733-2 & n.a. ${ }^{66}$ \\
\hline HEA125 & Human epithelium antigen ${ }^{67}$ \\
\hline $\mathrm{KS} 1 / 4$ & Carcinoma-associated glycoprotein ${ }^{65}$ \\
\hline MK-1 & n.a. ${ }^{6,68}$ \\
\hline MH99 & n.a. ${ }^{21,67}$ \\
\hline MOC31 & n.a. ${ }^{37}$ \\
\hline 323/A3 & n.a. ${ }^{69}$ \\
\hline $17-1 \mathrm{~A}$ & n.a. ${ }^{70}$ \\
\hline CO-17A & n.a. ${ }^{71}$ \\
\hline \multicolumn{2}{|c|}{ Based on a cDNA clone used to characterize the antigen } \\
\hline EpCAM/Ep-CAM & Epithelial cell adhesion molecule $e^{72}$ \\
\hline ESA & Epithelial surface antigen ${ }^{73}$ \\
\hline EGP-2 & Epithelial glycoprotein-2 $2^{74}$ \\
\hline EGP34 & Epithelial glycoprotein of $M_{r} 34,000^{26}$ \\
\hline EGP40 & Epithelial glycoprotein of $M_{r} 40,000^{75}$ \\
\hline KSA & Adenocarcinoma-associated antigen ${ }^{76}$ \\
\hline $\mathrm{KS} 1 / 4$ & n.a. ${ }^{67}$ \\
\hline TROP-1 & Trophoblast cell-surface antigen ${ }^{40}$ \\
\hline TACST-1 & Tumor-associated calcium signal transducer $1^{77}$ \\
\hline
\end{tabular}

n.a., not available.

single-spanning transmembrane domain and an intracellular domain containing an NPXY internalization motif and several $\alpha$-actinin binding sites. ${ }^{5}$

EpCAM was initially described as a tumor-associated antigen by Koprowski and colleagues ${ }^{6}$ in 1979. It is of particular interest because of its high level of expression on a variety of carcinomas. Thus, it comes as no surprise that EpCAM is a candidate protein for tumor diagnosis and therapy. Most studies have focused on EpCAM as a favorable target for tumor therapy, involving monoclonal and bi-/tri-specific antibodies, ${ }^{7}$ vaccination strategies, ${ }^{8}$ toxin-conjugated antibody fragments, ${ }^{9}$ and an antibody fragment-targeted STRAIL fusion protein. ${ }^{10}$

In contrast to the broad expression pattern of most other CAMs in normal tissues, the expression of EpCAM is restricted to normal epithelial cells. Based on the observation that EpCAM mediates cell-cell adhesion, Litvinov and colleagues ${ }^{11}$ proposed that EpCAM is a CAM. However, recent insights revealed a more versatile role for EpCAM, not merely limited to cell adhesion but similar to other CAMs, including processes such as signaling, cell migration, proliferation, and differentiation (Table 2).

Table 2. Functions Initially Proposed for EpCAM Based on Changes in Expression Pattern and Level and Binding Partners of EpCAM

Processes associated with EpCAM

Cell-cell adhesion

Cell migration

Metastasis

Cell differentiation

Cell proliferation

Morphogenesis:

organogenesis and regeneration

Cell signaling

Cell cycle Metabolism
- Evidenced by an ultrastructural localization of EpCAM ${ }^{11}$

- Lack of EpCAM affects aggregation of epithelial cells ${ }^{11}$

- Mediates interactions between intestinal epithelial cells and intraepithelial lymphocytes ${ }^{50}$

- An element of tight junctions via interaction with claudin- $7^{43}$

- In vitro knockout of EpCAM (siRNA) decreases migration rate ${ }^{51}$

- Interaction with $\alpha$-actinin ${ }^{38}$

- Interaction with CD44v4-v7 ${ }^{41}$ and CD948

- Disruption of EpCAM-mediated interactions, affects cell differentiation ${ }^{20,51}$

- Changes in expression level and pattern during epithelia differentiation 20

- In vitro knockout of EpCAM (siRNA) enables proliferation ${ }^{51}$

- Up-regulation of the proto-oncogene c-Myc and cyclins $A / E^{61}$

Involvement in:

- Pancreatic development ${ }^{20}$

- Kidney development (M.T., E.R. Popa, P.M.J.M., H. van Goor, A. Timmer, G.W. Bosman, L.F.M.H.d.L., and M.C.H., accepted)

- Liver regeneration ${ }^{16}$

- Kidney regeneration (M.T., P.M.J.M., H. van Goor, G. van Dam, L.F.M.H.d.L., E.R. Popa, and M.C.H., submitted)

- Up-regulation of the proto-oncogene c-Myc and cyclins $A / E^{61}$

- Reorganization of the actin cytoskeleton via interaction with $\alpha$-actinin ${ }^{38}$

- Up-regulation of the proto-oncogene c-Myc and cyclins $A / E^{61}$

- Regulation of expression of the epidermal fatty acid-binding protein (E-FABP) 62 

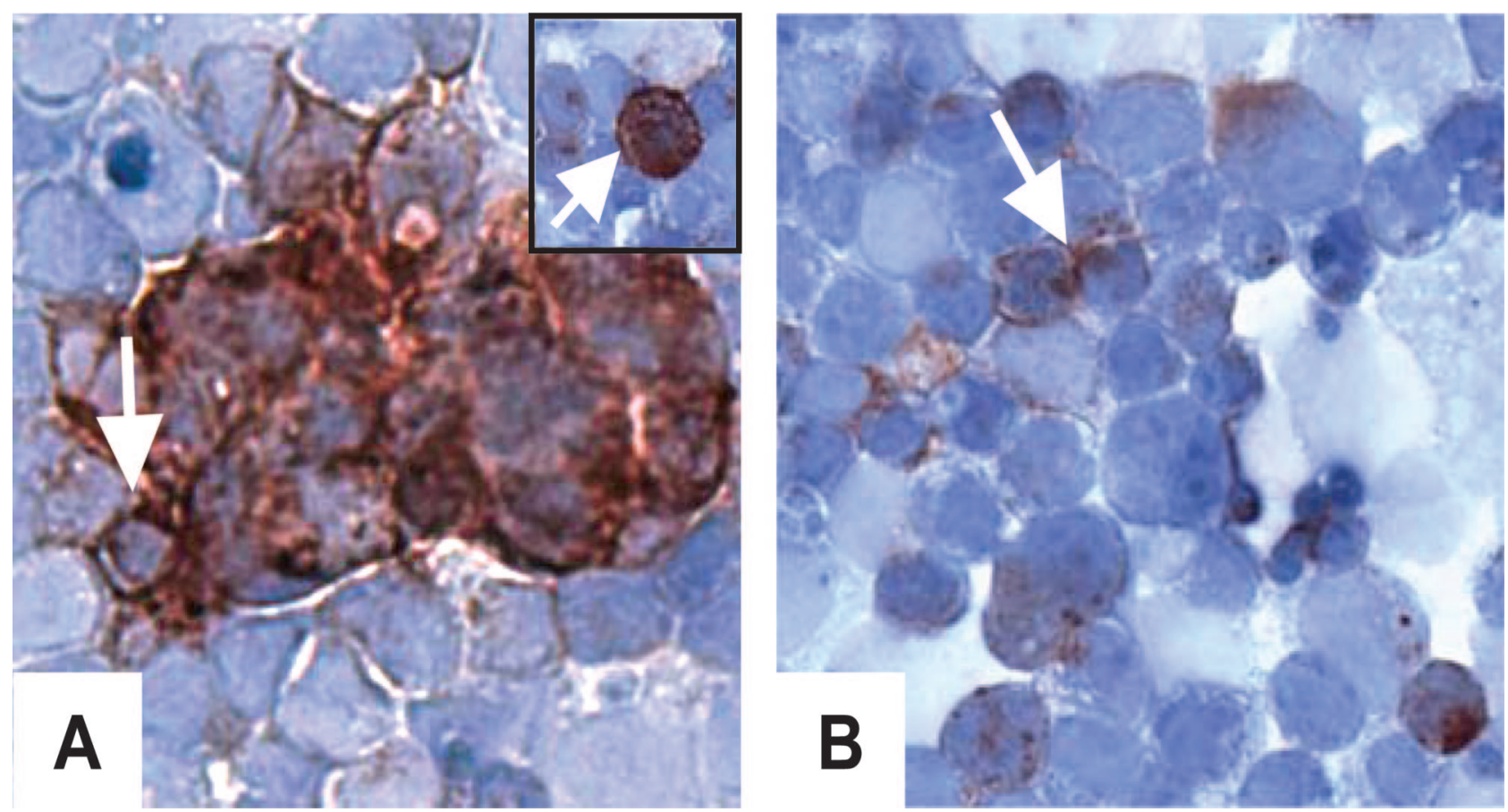

Figure 1. EpCAM expression pattern in murine EBs. A: EpCAM expression along the cell membrane (arrow) and intracellular expression (inset, arrow). B: EPCAM expression on these parts of the cell membrane, in contact with a neighboring cell (arrow). Staining intensity indicates that EpCAM expression level in EB differs between cells.

Here, we review the current knowledge of EpCAM biology in relation to other CAMs. We discuss the implications of the various newly identified functions of EpCAM in light of its prognostic factor in carcinoma, its use in anti-carcinoma therapy, and its expression on normal epithelia.

\section{Expression Patterns of EpCAM}

The expression pattern of CAMs is highly diverse, both between the different CAM families and within the families. However, most of the CAMs are present on virtually all cells. This is in contrast to the expression of EpCAM, which in healthy individuals is restricted to simple epithelia. Moreover, EpCAM is expressed in pathological situations, most notably in carcinoma but also in inflammatory diseases. Here, we detail the expression pattern and level of EpCAM during development and in normal healthy adult tissues as well as in pathological conditions, such as cancer and inflammatory disease.

\section{Expression of EpCAM in Embryogenesis and during Differentiation}

Embryonic development depends on the appropriate expression and interactions of CAMs. Most of the CAMs are expressed during embryogenesis from the earliest developmental stages in a tissue-specific way. Similarly, EpCAM is expressed in the first developmental stages, ie, in fertilized oocytes, at the two-cell stage and in the morula. $^{12}$
Moreover, expression of classic CAMs is found on embryonic stem cells, which is not surprising because cell-cell interactions are crucial for differentiation. Likewise, in murine embryonic stem cells, expression of the murine homologue of EpCAM has been found. ${ }^{13}$ Thus, although EpCAM expression is restricted to epithelia in adult tissues, EpCAM is also present in embryonic stem cells that are not yet committed to the epithelial cell type during embryonal development. This early embryonic expression of EpCAM on nonepithelial precursors is a feature that EpCAM shares with other CAMs throughout the different CAM families. In addition, we recently observed the early expression of murine EpCAM in embryoid bodies (EBs) in vitro (Figure 1). Interestingly, in the EB three phenotypically distinct populations of cells could be distinguished with regard to expression pattern of EpCAM (Figure 1). In the first population, EpCAM was expressed only intracellularly. In the second population, EpCAM was distributed homogeneously along the cell membrane whereas in the third population EpCAM expression was restricted to only those parts of the membrane that were in close contact with neighboring cells. No intracellular EpCAM could be found in a third population. A similar expression pattern has been found for E-cadherin ${ }^{14}$ and platelet endothelial cell adhesion molecule during differentiation of EBs. ${ }^{15}$ Such a distribution of EpCAM in EBs may be associated with the differentiation of these cells.

During embryogenesis, EpCAM expression is induced during the process of maturation and differentiation of some structures, for instance in comma- and S-shaped bodies in developing kidney (M.T., E.R. Popa, P.M.J.M., H. van Goor, A. Timmer, G.W. Bosman, L.F.M.H.d.L., and 
M.C.H., accepted). However, terminally differentiated, ie, specialized, adult epithelial cells typically cease to express EpCAM. For instance, small bile duct epithelium and bile canaliculi in the adult liver express EpCAM, whereas hepatocytes and large bile ducts are negative. ${ }^{16}$ Furthermore, in normal colonic crypts the germinal regions of the colonic mucosal lining display high EpCAM expression, which steadily decreases as cells differentiate and migrate toward the top of the villi. ${ }^{17}$ In addition, progenitor cells of skin epithelium express EpCAM, whereas differentiated keratinocytes do not. ${ }^{18}$ Such dynamic changes in expression pattern are typical not only for EpCAM. For instance, in developing kidney NCAM is expressed in S-shaped bodies and early tubules, whereas only minimal expression of NCAM is found in adult tubules. ${ }^{19}$

One of the classically ascribed functions of CAMs is their morphoregulatory role. In this respect, EpCAM expression has also been associated with morphogenesis based on its up-regulated expression in carcinomas and the marked variations in expression during development and regeneration of epithelia. ${ }^{16,20}$ Indeed, de novo expression of EpCAM was observed after induction of dedifferentiation in human keratinocytes that are normally EpCAM-negative. ${ }^{21}$ In addition, during culture and passage of human keratinocytes, significant increases in EpCAM expression were detected in parallel with a decrease of expression level of the differentiation marker BT15. ${ }^{21}$ Also, in squamous cervical epithelia EpCAM expression correlates with an enhanced proliferative activity and with the loss of tissue-specific markers, including markers for terminal differentiation of squamous epithelial cells. ${ }^{22}$ Taken together, in the initial phases of morphogenesis, EpCAM is found not only on epithelial precursor cells but also on cells that are not yet assigned to a certain cell fate. In later stages of epithelial development, EpCAM acquires a strictly epithelial-specific expression whereas in terminally differentiated cells EpCAM is not expressed.

\section{Expression of EpCAM in Mature Tissue}

In mature tissue, EpCAM is expressed in virtually all simple epithelia, and its expression is usually not subject to change. However, the expression of EpCAM can change in epithelial progenitor cells, which are required for tissue maintenance and turnover. For instance, bipotent progenitor cells of adult human mammary tissue as well as progenitor cells expressing luminal cell markers are both EpCAM-positive. These progenitors can give rise to progenitor myoepithelial cells, which do not express EpCAM. ${ }^{23}$ Interestingly, the loss of EpCAM correlates with a decrease in progenitor activity, ${ }^{23}$ which suggests that the level of cellular differentiation and specialization at least partly depends on EpCAM expression.

In contrast to the epithelia-restricted expression of human EpCAM, the murine homologue of EpCAM is expressed not only on epithelia but also on thymocytes, $T$ cells, and antigen-presenting cells. Several studies have indicated that the selective expression of CAMs on intraepithelial lymphocytes is important for the interaction of these lymphocytes with epithelial cells. ${ }^{24}$ Thus, this nonepithelial expression of murine EpCAM may contribute to homotypic adhesive interactions between thymocytes and dendritic cells or epithelial cells, as also described for the lymphocyte endothelial and epithelial CAM (LEEPCAM) or the integrin $\alpha \mathrm{E} \beta 7$-E-cadherin complex. ${ }^{24}$ Since the up-regulation of murine EpCAM in mature thymocytes is one of the sequels of activation in vitro, Nelson and colleagues $^{25}$ postulated that EpCAM is involved in thymocyte development and differentiation or peripheral Tcell trafficking and function.

\section{Expression of EpCAM in Carcinoma}

Aberrant expression, such as up-regulation, down-regulation, or de novo expression, is characteristic for most CAMs during and after malignant transformation. EpCAM is typically overexpressed in a variety of epithelial cancers, ie, carcinomas. EpCAM is not expressed on tumors of mesodermal and ectodermal origin, such as neurogenic tumors, sarcomas, melanomas, or lymphomas. ${ }^{26}$ Its overexpression usually correlates with a decrease in survival, for instance in breast and ovarian cancer. ${ }^{27,28}$ An exception is renal cell carcinoma, in which EpCAM expression correlates with an increase in life expectancy. ${ }^{29}$

In contrast to normal epithelia, in which EpCAM is expressed mostly on the basal or basolateral cell membrane, EpCAM distribution varies in carcinoma, depending on the type of carcinoma. For instance in colon carcinoma well-differentiated adenocarcinoma cells are characterized by a homogeneous basolateral distribution of EpCAM, whereas moderately differentiated adenocarcinoma cells typically have a membranous, cytoplasmic, and luminal EpCAM expression. ${ }^{30}$ More recently, Xie and colleagues $^{31}$ have provided further evidence that EpCAM is distributed differently in normal and various malignant colon tissues, including squamous cell carcinoma, signet-ring cell carcinoma, and adenocarcinomas. These observations may have a diagnostic value in the correct recognition of carcinoma type.

\section{Expression of EpCAM in Response to Inflammation}

Inflammatory responses require adhesive interactions between different populations of cells, for instance for the infiltration of immune effector cells into the site of inflammation, and CAMs are crucial components in this process. Furthermore, CAMs are required for the induction of processes that accompany tissue regeneration after inflammatory damage, ie, cell proliferation and differentiation. Consequently, most CAMs are up-regulated or de novo expressed during and after tissue inflammation, with the exception of E-cadherin, which is down-regulated during tissue regeneration. ${ }^{32}$

In contrast to E-cadherin, but in agreement with other CAMs, EpCAM is up-regulated during inflammatory responses, for example during severe mucosal inflammation in the liver. ${ }^{33}$ Moreover, hepatocytes that are normally EpCAM-negative, de novo express EpCAM during 


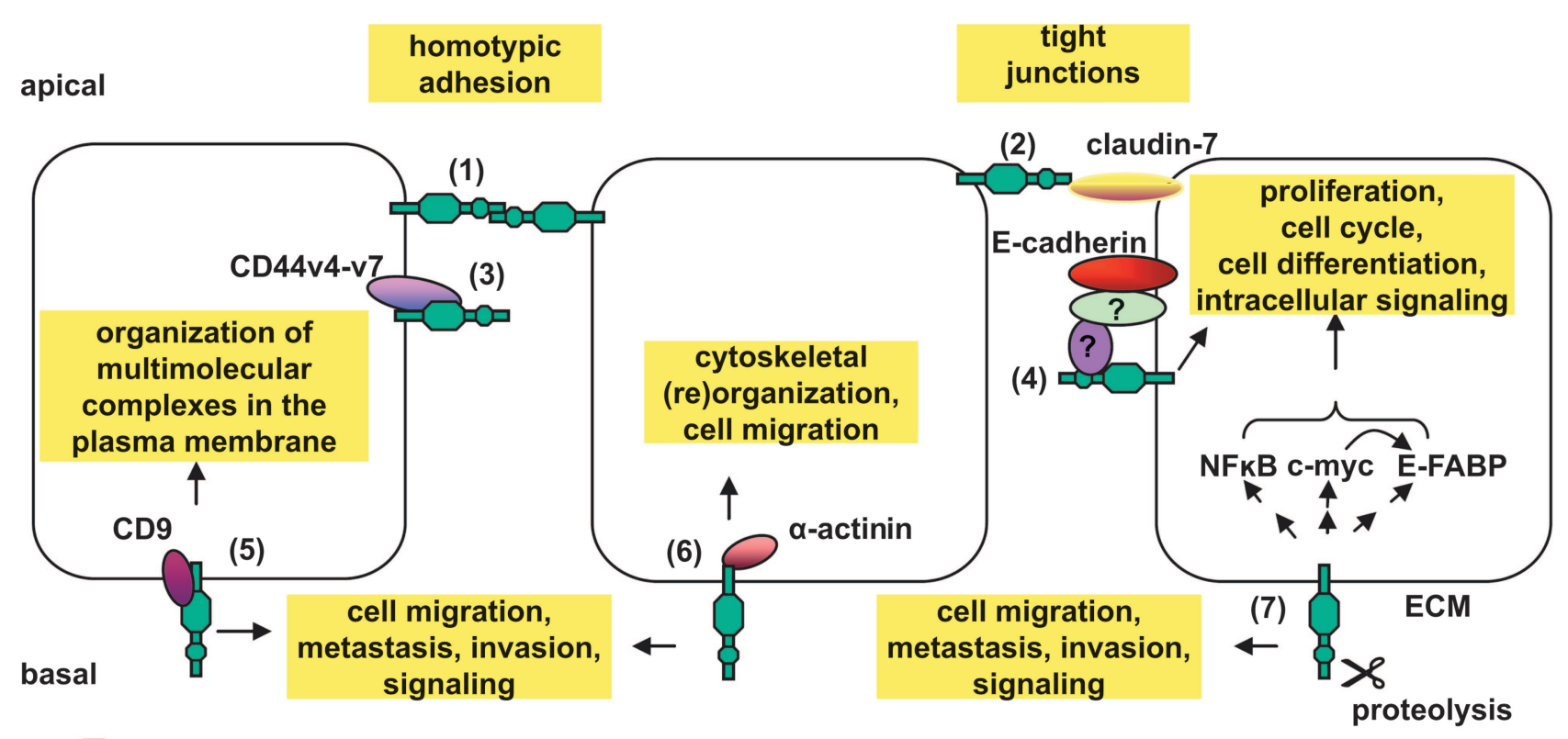

EpCAM

Figure 2. Schematic representation of ligands identified for EpCAM and possible functions related to them. (1) The extracellular domain of EpCAM interacts with a second EpCAM molecule resulting in homotypic cell-cell adhesion, hence, the name epithelial cell adhesion molecule. (2) EpCAM directly interacts with claudin-7, a protein required for the formation of tight junctions, again implying a role of EpCAM in cellular adherence. (3) EpCAM directly interacts with CD44v4-v7 (carcinomaassociated variants of CD44). The exact consequence of this interaction remains to be elucidated. (4) EpCAM indirectly associates with E-cadherin. The adaptor proteins that facilitate this complex formation have yet to be identified. (5) EpCAM forms a primary complex with CD9 in the tetraspanin web. (6) Direct interactions of $\alpha$-actinin with the intracellular domain of EpCAM links this molecule to the cytoskeletal organization. (7) EpCAM modulates the expression of NF- $\kappa$ B, c- $m y c$, and E-FABP. The molecular pathway of this modulation has not yet been found. We propose that a proteolytic cleavage in the extracellular domain may initiate a signaling cascade that results in the augmented expression of NF- $\kappa \mathrm{B}, \mathrm{c}-m y c$, and E-FABP. The interplay of EpCAM with E-cadherin (4) and CD9 (5), as well as the influence of EpCAM on NF- $\kappa \mathrm{B}$ c- $m y c$, and E-FABP (7) provides a link for its involvement in cell migration, metastasis, and cell signaling. ECM, extracellular matrix; E-FABP, epidermal fatty acid-binding protein.

chronic necroinflammatory diseases. ${ }^{33}$ In contrast, primary sclerosing diseases are characterized by downregulation of EpCAM expression. ${ }^{33}$ Thus, the de novo expression of EpCAM is probably also a part of an inflammatory response and might be linked to regenerative activity and (re-)epithelization in response to inflammation. In this respect, it has previously been shown that epithelial damage induces the up-regulation of EpCAM in regenerating areas of the liver. ${ }^{16}$ Recently, we found EpCAM expression to be up-regulated in renal cortex after ischemic damage (M.T., E.R. Popa, P.M.J.M., H. van Goor, G. van Dam, L.F.M.H.d.L., E.R. Popa, and M.C.H., submitted). Thus, the expression level of EpCAM changes in regenerating epithelia.

\section{Regulation of EpCAM Expression}

To date, little is known about the molecular mechanisms that govern the pattern and level of EpCAM expression. One step forward toward deciphering this molecular mechanism was recently achieved by Gires and colleagues, ${ }^{34}$ who showed that tumor necrosis factor (TNF)- $\alpha$ can down-regulate EpCAM. This down-regulation coincides with the activation of nuclear factor (NF)- $\kappa \mathrm{B}$ and is mediated by a signaling cascade that involves the TNF receptor 1 (TNF-R1) and the direct cytoplasmic ligand of TNF-R1, TNFR1-associated death domain protein (TRADD). However, the negative regulation led by TNF- $\alpha$ is in contradiction to the abovedescribed de novo expression of EpCAM in chronic inflammatory liver diseases. In addition, Flieger and colleagues ${ }^{35}$ reported a modulating effect of TNF- $\alpha$ on EpCAM expression, which might be a link between de novo EpCAM or up-regulated expression level of EpCAM in epithelial cells under inflammatory conditions. In carcinoma, chemotherapeutic agents such as vinorelbine tartrate (Navelbine, GlaxoSmithKline, Philadelphia, PA) and paclitaxel (Taxol; Bristol Myers Squibb, Princeton, NJ) have also been shown to modulate EpCAM expression. ${ }^{36}$ Because paclitaxel is known to modulate NF- $\kappa$ B activity, it is tentative to speculate that the effect of these chemotherapeutics may be attributable to the NF- $\kappa \mathrm{B}$-mediated regulation of EpCAM promoter activity.

\section{The Pleiotropic Nature of EpCAM}

\section{Binding Partners of CAMs}

The extracellular domains of CAMs contain regions that bind proteins of the extracellular matrix, including collagen, laminin, and fibronectin, as well as other integral cell-surface plasmalemmal proteins. The cytoplasmic domains comprise regions that bind to cytoskeletal elements, for instance talin, vinculin, and $\alpha$-actinin. In the following section the interaction of EpCAM with several ligands, all of which are themselves involved in various regulatory processes, will be reviewed (Table 2 and Figure 2). 


\section{Early Reports on Binding Partners of EpCAM}

The first binding partner of EpCAM reported in literature was a protein of $30 \mathrm{kd}$ found in EpCAM-expressing carcinoma cells. ${ }^{37}$ Helfrich and colleagues ${ }^{37}$ used recombinant soluble EpCAM as a probe for the EpCAM-binding protein. Later, Schön and colleagues ${ }^{21}$ reported that the extracellular domain of EpCAM is covalently bound to a protein of a high molecular weight, which is located in the extracellular matrix. However, the identity of these EpCAM-binding proteins has not been revealed yet.

\section{Interaction of EPCAM with the Actin Cytoskeleton}

The cytoplasmic intracellular domain of EpCAM contains two $\alpha$-actinin binding sites, through which EpCAM interacts with $\alpha$-actinin and, consequently, with the actin cytoskeleton. $^{38}$ In vitro, ectopic expression of EpCAM in thymic stromal cells, which are normally EpCAM-negative, results in a rapid reorganization of the actin cytoskeleton. In this process, numerous stress fibers as well as long cellular protrusions are formed. ${ }^{38}$ Simultaneously, E-cadherin-dependent cell adhesion is destabilized. Moreover, ectopic overexpression of EpCAM leads to disorganization of epithelial cell layers and enhances growth of epithelia. Based on these in vitro data, Guillemot and colleagues ${ }^{38}$ proposed that EpCAM determines the microenvironment in the thymic epithelium. Interestingly, in cells characterized by a disorganized cytoskeleton, eg, rat metastasizing pancreatic adenocarcinoma cells, EpCAM mediates stronger adhesions when compared to cells with a more organized cytoskeleton, ie, non/low-metastasizing pancreatic adenocarcinoma. ${ }^{39}$ Therefore, Wurfel and colleagues ${ }^{40}$ proposed that in the absence of an organized cytoskeleton, the adhesive functions of EpCAM take over a more dominant role. These data show that EpCAM participates in the formation and regulation of a dynamic stromal cell network in vitro and thus, like other CAMs, may be involved in these processes in vivo.

\section{Association of EpCAM with CD44V4-V7 and Claudin-7}

Recently, EpCAM was shown to interact directly with CD44v4-v7, ${ }^{41}$ a tumor metastasis-promoting CAM, ${ }^{42}$ and with claudin-7, a tight junction protein. ${ }^{43}$ In complexes of CD44v4-v7-tetraspanin-EpCAM-claudin-7, found in pancreatic tumor cell lines, the intracellular domain of EpCAM directly interacted with CD44v4-v7. In addition, the extracellular domain of EpCAM interacted with claudin-7. Interestingly, although EpCAM interacts with CD44v4-v7, it does not interact with the standard CD44 isoform.

These carcinoma-specific complexes of EpCAM with CD44v4-v7 and claudin-7 can influence cell-cell adhesion, cell-matrix adhesion, and apoptosis resistance, and they appear to be involved in processes that promote carcinogenesis, ie, metastasis. ${ }^{41}$ The EpCAM-claudin-7 complex is frequently observed in highly metastatic car- cinomas, such as pancreatic and colorectal. Furthermore, as described above, EpCAM overexpression is associated with poor prognosis in pancreatic and colorectal carcinomas. Therefore, Ladwein and colleagues ${ }^{43}$ suggested that these complexes support tumor progression by promoting metastasis. This notion is supported by the fact that one of the other members of the complex, CD44v4-v7, is a surface molecule that promotes tumor metastasis. ${ }^{42}$ On the other hand, during invasion and metastasis in breast and esophageal carcinoma, the expression level of claudin-7 is reduced. ${ }^{44-46}$ Furthermore, claudin-7 is a crucial element of tight junctions ${ }^{43}$ and, therefore, may prevent invasion and metastasis by keeping the tissue compact. Interestingly, although ultrastructural analysis of EpCAM-mediated adhesions did not reveal any junctional contacts, ${ }^{5}$ EpCAM may be associated with formation of tight junction and/or its maintenance by the direct interaction between claudin-7 and EpCAM.

\section{EpCAM in the Tetraspanin Web}

Tetraspanins are integral membrane proteins, clustered in specific tetraspanin-enriched microdomains that regulate the functions of associated transmembrane proteins, including CAMs. A small fraction of total cell surfaceexpressed EpCAM is associated with the tetraspanin web via its interaction with D6.1A, a protein that itself is found in the tetraspanin D6.1A/CO-029/TM4SF3 complex. As such, EpCAM appears to be recruited to tetraspanin-enriched microdomains. ${ }^{47}$ Since tetraspanins have been associated with metastasis, it is tentative to speculate that the potential prometastatic function of EpCAM is a consequence of its association with tetraspanin-enriched microdomains. Corroborating evidence for the association of EpCAM with tetraspaninenriched microdomains has come from a recent report in which proteome analysis of the tetraspanin web in colon cancer revealed that EpCAM is part of the tetraspanin web in a newly identified primary complex with CD9. ${ }^{48}$

\section{Homotypic Interaction of EpCAM in Mucosal Defense against Infection}

CAMs are used by intraepithelial lymphocytes to adhere and function in intestinal epithelia cells. ${ }^{49}$ Such a direct interaction of intestinal epithelia cells and intraepithelial lymphocytes provides an immunological barrier against mucosal infection. Recently, EpCAM was found to mediate homotypic interactions between intraepithelial lymphocytes and intestinal epithelia cells. ${ }^{50}$ Consequently, one might anticipate that EpCAM could be involved in the immune response against mucosal infections. However, further studies are required to confirm this hypothesis.

\section{The Double Face of EpCAM: EpCAM in Cell Adhesion, Migration, and Metastasis}

Typically, CAMs are responsible for proper cell-cell interactions, cell polarity, and tissue integrity. As such, CAMs 
are at the basis of various processes such as cell proliferation, differentiation, signaling, mesenchymal to epithelial transition, as well as the opposite process, epithelial to mesenchymal transition. However, it is interesting to note that EpCAM lacks the multiple repeated subdomains characteristic for CAMs of the known subfamilies of cadherins, integrins, selectins, and CAMs of the immunoglobulin superfamily. ${ }^{5}$ Thus, the tertiary structure of EpCAM does not resemble that of other adhesion molecules.

Initially, Litvinov and colleagues ${ }^{11}$ proposed EpCAM to be a calcium-independent homotypic adhesion molecule based on their observation that nonadhesive cancer cells acquired adhesive properties and formed aggregates on ectopic overexpression of EpCAM. Corroborating evidence for the role of EpCAM as homophilic CAM has been obtained in studies on its role in mucosal defense, as described above. ${ }^{50}$ However, it appears that EpCAM might have an opposite role to the classic CAMs. Litvinov and colleagues ${ }^{11}$ reported that EpCAM is a functional antagonist to E-cadherin. More recent studies showed that EpCAM negatively modulates cadherin-mediated cell adhesion by disruption of the link between $\alpha$-catenin and F-actin. In this way, EpCAM loosens the tight cell-cell adhesions and modulates proliferation, differentiation, and tissue maintenance. ${ }^{51,52}$ As such, it might be anticipated that overexpression of EpCAM, typical for carcinoma cell lines, is an important mechanism to disrupt cell-cell contact to enable cell migration required for metastasis. Thus, EpCAM may be a prometastatic molecule. A role for EpCAM during metastasis was also confirmed in vivo for the rat ortholog of EpCAM, D5.7A. ${ }^{39}$ Wurfel and colleagues $^{39}$ injected rats with normally low metastasizing tumors, eg, fibrosarcomas, that were engineered to ectopically overexpress D5.7A. Only on overexpression of D5.7A were tumor cells found to metastasize to the distal lymph nodes and the lung. ${ }^{39}$ These findings are further corroborated by the fact that in human cancer patients elevated EpCAM expression is linked to an increase in lymph node metastases. ${ }^{53}$ Moreover, on downregulation of EpCAM using siRNA in renal and breast carcinoma cell lines, cell migration does not occur in the absence of EpCAM. ${ }^{29,51}$ Together, this clearly links expression of EpCAM to formation of metastases.

Under physiological conditions, EpCAM may have a similar role in promoting migration. For instance, EpCAM is highly expressed in colon and hair follicles, where epithelial precursor cells move along the villus to reach the top. ${ }^{17}$ This migratory process is primarily reminiscent of metastasis, thus lending further credit for the importance of EpCAM in the formation of metastasis.

In contrast, in human tumor cells that undergo epithelial to mesenchymal transition, when the epithelial cells acquire mesenchymal features to locomote, EpCAM expression is transiently down-regulated to enable migration. ${ }^{54}$ Similarly, during structural rearrangements and cell migration taking place in nephrogenesis, when low intercellular adhesions are preferable, EpCAM expression level is also low (M.T., E.R. Popa, P.M.J.M., H. van Goor, A. Timmer, G.W. Bosman, L.F.M.H.d.L., and M.C.H., accepted). This phenomenon seems to contra- dict the correlation between EpCAM and cell migration processes.

Furthermore, double function of EpCAM might result from the fact that the extracellular domain of EpCAM contains a proteolytic cleavage. Such a cleavage of the extracellular domain of EpCAM can regulate cells adhesiveness/nonadhesiveness. Wurfel and colleagues ${ }^{39}$ showed that protease treatment of the rat-derived pancreatic adenocarcinoma cell line strengthened cell-cell adhesion and significantly increased the proliferative activity of EpCAM. A similar regulatory mechanism of proteolytic cleavage of the extracellular domain and release of the extracellular domain has been shown to be of major importance for the regulation of cell adhesion guided by many other CAMs. For instance, proteolytic cleavage of the extracellular domain of $\mathrm{N}$ - and $\mathrm{E}$-cadherin decreases the adhesiveness of cells. ${ }^{55}$ Furthermore, without proteolytic cleavage of $\alpha(\mathrm{v})$ integrins, their function in cell adhesions is altered. ${ }^{56}$ Moreover, cleavage of the CD44 ectodomain plays a critical role in CD44-mediated tumor cell migration by regulating the dynamic interaction between CD44 and the extracellular matrix. ${ }^{57}$

Of note, glycosylation is a highly diverse nontemplatedriven process that can generate enormous informational content. In this respect, the epidermal growth factor-like repeat in the extracellular domain of $\mathrm{EpCAM}^{4}$ may be a target for differential glycosylation and, therefore, the choice of specific binding partner of EpCAM, and may consequently regulate the EpCAM-mediated adhesiveness. It is well established that the glycosylation pattern of EpCAM markedly differs between carcinomas and normal epithelia, as well as between different carcinomas. ${ }^{58}$ It is interesting to determine whether the positive effect of EpCAM on patient survival in renal cell carcinoma, in contrast to the negative effect on survival of EpCAM in many other carcinomas, is related to a difference in glycosylation pattern, subsequent signaling, cell migration, or cell adhesion.

Thus, it seems that depending on the microenvironment, as well as the EpCAM expression level, EpCAM supports adhesion in normal epithelia, whereas in carcinoma EpCAM can switch between two functions. In the first situation, EpCAM prevents strong cell-cell adhesion and thereby enables cell migration and metastasis, similarly to E-cadherin. In the second situation, EpCAM mediates cell-cell adhesion and thereby prevents cell migration.

Such a double function was reported also for CD44, another CAM, in neoplasia. CD44 can act as a growth/ invasion-promoting molecule or a tumor suppression co-factor. ${ }^{59}$ Herrlich and colleagues ${ }^{59}$ proposed that the type of action of CD44 on a given cell depends, among others, on the nature of the extracellular matrix and on the interactions with different proteins that link plasma membrane and the actin-containing cytoskeleton. This can be also an explanation for the double face of EpCAM, because EpCAM can also interact with ECM and cytoskeleton via functionally different proteins. 


\section{EpCAM in Cell Proliferation}

Studies on the rat ortholog of EpCAM, D5.7A, also revealed that cross-linking of D5.7A provides a proliferative signal for carcinoma cells. ${ }^{39}$ Furthermore, Osta and colleagues ${ }^{51}$ showed that down-regulation of EpCAM using siRNA leads not only to a decrease in migration but also to a decrease in cell proliferation in breast carcinoma. These data indicate that EpCAM, like other CAMs, is required for cell proliferation and differentiation in carcinoma, which, although not proven, might also be the case for normal epithelial cells. However, down-regulation of EpCAM only reduces but does not abolish proliferation and migration. Therefore, we suggest that although EpCAM is clearly involved in these processes, other proteins are also involved in regulating proliferation and migration.

\section{EpCAM in Cell Cycle Regulation}

Already in 1990 Simon and colleagues $^{60}$ proposed a receptor-like function for EpCAM, based on the high DNA sequence homology of EpCAM to nidogen and placental protein 12, a matrix adhesion molecule and an insulin-like growth factor-binding protein, respectively. More recent evidence presented by Munz and colleagues, ${ }^{61}$ has refined the effect of EpCAM to cell cycle regulation. In their studies, Munz and colleagues ${ }^{61}$ ectopically expressed the intracellular domain of EpCAM in the EpCAM-negative human embryonic kidney cell line HEK293. This ectopic expression resulted in the up-regulation of c-myc as well as cyclin $A$ and $E .{ }^{61}$ The net effect was enhanced cell cycle progression and a resultant increase in proliferation and metabolism. ${ }^{61}$

In addition, the epidermal fatty acid-binding protein (E-FABP) was recently identified as another gene indirectly regulated by EpCAM. ${ }^{62}$ E-FABP expression is rapidly up-regulated on induction of EpCAM in squamous cell carcinoma lines and in primary head and neck carcinomas in vivo. Of note, E-FABP has been reported to be a target gene of $\mathrm{c}-\mathrm{Myc}$, thus providing a potential link between EpCAM-induced c-Myc expression and E-FABP expression. These results provide evidence for the direct involvement of EpCAM in signaling processes, gene regulation, and cellular metabolism and support an important role of EpCAM in tumor biology, as well as in normal epithelial cells.

Based on the diverse functions of the various binding partners of EpCAM (Table 2) we propose that the local microenvironmental circumstances set the stage for the different functions of EpCAM. In other words, the activity of EpCAM is probably governed by the selective binding to its respective partners and other associated molecules that are located either at the cell membrane or in the cytoplasm.

\section{Summary and Perspectives}

Throughout the past 4 decades, the expression of EpCAM has been investigated predominantly in malignant tissues for diagnostic and prognostic purposes. As a consequence, a variety of therapeutic strategies have been designed that specifically target EpCAM based on its overexpression in carcinomas. However, EpCAM is more than a carcinoma marker. Although its structure does not resemble any of the classical CAMs, EpCAM has been proposed to be a CAM molecule because it can mediate cell-cell adhesion. Recently, the position of EpCAM as a CAM was strengthened by the observation that EpCAM, besides adhesion, participates in other CAMspecific processes that underlie morphogenesis and metastases, such as proliferation, differentiation, migration cell-cycle regulation, and intracellular signaling. Thus, EpCAM clearly is a pleiotropic molecule that serves an important role in the onset, development, maintenance, repair, and function of epithelia in the organism.

Most of the studies dedicated to the function of EpCAM come from in vitro studies. The most obvious way to elucidate further the exact role of EpCAM is by generating a knockout model. However, because EpCAM is expressed at very early developmental stages and CAMbased adhesions and junctions are crucial for the mammalian epidermal barrier, ${ }^{63}$ it is to be expected that a knockout model is embryonic lethal. Therefore, conditional knockouts dedicated to a particular embryonic stage or epithelial organ might provide the best way forward and allow us to identify, at which stage during development, during tissue repair, or during tumor metastases EpCAM is critical. To elucidate the role of EpCAM in carcinoma, it would be interesting to develop a knockin model.

The various roles of EpCAM can also have profound implications for carcinoma therapy. In certain situations, blocking EpCAM using monoclonal antibodies may not only diminish tumor growth but also prevent the formation of metastasis. In addition, since EpCAM promotes cell proliferation and, therefore, growth of the tumor, it seems to be worthwhile to combine EpCAM-targeted therapy with selective anti-proliferative agents such as paclitaxel or vinoreline.

In carcinoma, the up-regulated expression of EpCAM may be considered as a factor that disturbs the regulatory balance and facilitates aberrant cellular proliferation and differentiation. In contrast, in normal epithelia EpCAM expression provides the flexible plasticity that is required for normal epithelial development and maintenance. This is accomplished because EpCAM is involved in processes underlying morphogenesis. ${ }^{29}$ Therefore, EpCAM targeted therapies might be of interest to improve the regeneration of epithelial tissues, eg, after ischemia. This would augment (re-)epithelization and recovery. Although selective activation of EpCAM expression in normal tissue is still far from feasible, detailed examination of regulatory mechanisms that guide promoter activity will in the future help to identify strategies to specifically induce EpCAM expression.

In conclusion, many reports that point to the pleiotropic nature of EpCAM have recently appeared. Although much remains to be elucidated, the ongoing research efforts on EpCAM have paved the way toward an understanding of EpCAM biology. 


\section{Acknowledgment}

We thank Dr. E. Bremer (University of Groningen, University Medical Center, Groningen, The Netherlands) for helpful suggestions.

\section{References}

1. Okegawa T, Pong RC, Li Y, Hsieh JT: The role of cell adhesion molecule in cancer progression and its application in cancer therapy. Acta Biochim Pol 2004, 51:445-457

2. Petruzzelli L, Takami M, Humes HD: Structure and function of cell adhesion molecules. Am J Med 1999, 106:467-476

3. Maheshwari G, Brown G, Lauffenburger DA, Wells A, Griffith LG: Cell adhesion and motility depend on nanoscale RGD clustering. J Cell Sci 2000, 113:1677-1686

4. Baeuerle PA, Gires O: EpCAM (CD326) finding its role in cancer. Br J Cancer 2007, 96:417-423

5. Balzar M, Winter MJ, de Boer CJ, Litvinov SV: The biology of the 17-1A antigen (Ep-CAM). J Mol Med 1999, 77:699-712

6. Koprowski H, Steplewski Z, Mitchell K, Herlyn M, Herlyn D, Fuhrer P: Colorectal carcinoma antigens detected by hybridoma antibodies. Somatic Cell Genet 1979, 5:957-971

7. Riesenberg R, Buchner A, Pohla $H$, Lindhofer $H$ : Lysis of prostate carcinoma cells by trifunctional bispecific antibodies (alpha EpCAM $x$ alpha CD3). J Histochem Cytochem 2001, 49:911-917

8. Mosolits S, Markovic K, Frodin JE, Virving L, Magnusson CG, Steinitz M, Fagerberg J, Mellstedt $\mathrm{H}$ : Vaccination with Ep-CAM protein or anti-idiotypic antibody induces Th1-biased response against $\mathrm{MHC}$ class I- and II-restricted Ep-CAM epitopes in colorectal carcinoma patients. Clin Cancer Res 2004, 10:5391-5402

9. Di Paolo C, Willuda J, Kubetzko S, Lauffer I, Tschudi D, Waibel R, Pluckthun A, Stahel RA, Zangemeister-Wittke U: A recombinant immunotoxin derived from a humanized epithelial cell adhesion molecule-specific single-chain antibody fragment has potent and selective antitumor activity. Clin Cancer Res 2003, 9:2837-2848

10. Bremer E, Kuijlen J, Samplonius D, Walczak H, de Leij L, Helfrich W: Target cell-restricted and -enhanced apoptosis induction by a scFv: STRAIL fusion protein with specificity for the pancarcinoma-associated antigen EGP2. Int J Cancer 2004, 109:281-290

11. Litvinov SV, Balzar M, Winter MJ, Bakker HA, Briaire-de Bruijn $I H$, Prins F, Fleuren GJ, Warnaar SO: Epithelial cell adhesion molecule (Ep-CAM) modulates cell-cell interactions mediated by classic cadherins. J Cell Biol 1997, 139:1337-1348

12. Tarmann T, Dohr G, Schiechl H, Barth S, Hartmann M: Immunohistochemical detection of an epithelial membrane protein in rat embryos at different stages of development. Acta Anat (Basel) 1990, 137:141-145

13. Anderson R, Schaible K, Heasman J, Wylie C: Expression of the homophilic adhesion molecule, Ep-CAM, in the mammalian germ line. J Reprod Fertil 1999, 116:379-384

14. Choi D, Lee HJ, Jee S, Jin S, Koo SK, Paik SS, Jung SC, Hwang SY, Lee KS, Oh B: In vitro differentiation of mouse embryonic stem cells: enrichment of endodermal cells in the embryoid body. Stem Cells 2005, 23:817-827

15. Redick SD, Bautch VL: Developmental platelet endothelial cell adhesion molecule expression suggests multiple roles for a vascular adhesion molecule. Am J Pathol 1999, 154:1137-1147

16. de Boer CJ, van Krieken JH, Janssen-van Rhijn CM, Litvinov SV: Expression of Ep-CAM in normal, regenerating, metaplastic, and neoplastic liver. J Pathol 1999, 188:201-206

17. Schiechl H, Dohr G: Immunohistochemical studies of the distribution of a basolateral-membrane protein in intestinal epithelial cells (GZ1$\mathrm{Ag}$ ) in rats using monoclonal antibodies. Histochemistry 1987, $87: 491-498$

18. Klein CE, Cordon-Cardo C, Soehnchen R, Cote RJ, Oettgen HF, Eisinger M, Old LJ: Changes in cell surface glycoprotein expression during differentiation of human keratinocytes. J Invest Dermatol 1987, 89:500-506

19. Abbate M, Brown D, Bonventre JV: Expression of NCAM recapitulates tubulogenic development in kidneys recovering from acute ischemia. Am J Physiol 1999, 277:F454-F463
20. Cirulli V, Crisa L, Beattie GM, Mally MI, Lopez AD, Fannon A, Ptasznik A, Inverardi L, Ricordi C, Deerinck T, Ellisman M, Reisfeld RA, Hayek A: KSA antigen Ep-CAM mediates cell-cell adhesion of pancreatic epithelial cells: morphoregulatory roles in pancreatic islet development. J Cell Biol 1998, 140:1519-1534

21. Schön MP, Limat A, Hartmann B, Klein CE: Characterization of an 80-kD membrane glycoprotein (gp80) of human keratinocytes: a marker for commitment to terminal differentiation in vivo and in vitro. J Invest Dermatol 1995, 105:418-425

22. Litvinov SV, van Driel W, van Rhijn CM, Bakker HA, van Krieken $H$, Fleuren GJ, Warnaar SO: Expression of Ep-CAM in cervical squamous epithelia correlates with an increased proliferation and the disappearance of markers for terminal differentiation. Am J Pathol 1996, 148:865-875

23. Stingl J, Eaves CJ, Zandieh I, Emerman JT: Characterization of bipotent mammary epithelial progenitor cells in normal adult human breast tissue. Breast Cancer Res Treat 2001, 67:93-109

24. Agace WW, Higgins JM, Sadasivan B, Brenner MB, Parker CM T-lymphocyte-epithelial-cell interactions: integrin $\alpha(E)(C D 103) \beta(7)$, LEEP-CAM and chemokines. Curr Opin Cell Biol 2000, 12:563-568

25. Nelson AJ, Dunn RJ, Peach R, Aruffo A, Farr AG: The murine homolog of human Ep-CAM, a homotypic adhesion molecule, is expressed by thymocytes and thymic epithelial cells. Eur J Immunol 1996, 26:401-408

26. Momburg F, Moldenhauer G, Hammerling GJ, Moller P: Immunohistochemical study of the expression of a $M_{r}$ 34,000 human epitheliumspecific surface glycoprotein in normal and malignant tissues. Cancer Res 1987, 47:2883-2891

27. Spizzo G, Went $P$, Dirnhofer S, Obrist $P$, Simon R, Spichtin H, Maurer R, Metzger U, von Castelberg B, Bart R, Stopatschinskaya S, Kochli OR, Haas P, Mross F, Zuber M, Dietrich H, Bischoff S, Mirlacher M, Sauter G, Gastl G: High Ep-CAM expression is associated with poor prognosis in node-positive breast cancer. Breast Cancer Res Treat 2004, 86:207-213

28. Spizzo G, Went P, Dirnhofer S, Obrist P, Moch H, Baeuerle PA, Mueller-Holzner E, Marth C, Gastl G, Zeimet AG: Overexpression of epithelial cell adhesion molecule (Ep-CAM) is an independent prognostic marker for reduced survival of patients with epithelial ovarian cancer. Gynecol Oncol 2006, 103:483-488

29. Seligson DB, Pantuck AJ, Liu X, Huang Y, Horvath S, Bui MH, Han KR, Correa AJ, Eeva M, Tze S, Belldegrun AS, Figlin RA: Epithelial cell adhesion molecule (KSA) expression: pathobiology and its role as an independent predictor of survival in renal cell carcinoma. Clin Cancer Res 2004, 10:2659-2669

30. Ogura E, Senzaki H, Yoshizawa K, Hioki K, Tsubura A: Immunohistochemical localization of epithelial glycoprotein EGP-2 and carcinoembryonic antigen in normal colonic mucosa and colorectal tumors. Anticancer Res 1998, 18:3669-3675

31. Xie X, Wang CY, Cao YX, Wang W, Zhuang R, Chen LH, Dang NN, Fang L, Jin BQ: Expression pattern of epithelial cell adhesion molecule on normal and malignant colon tissues. World J Gastroenterol 2005, 11:344-347

32. Zbar AP, Simopoulos C, Karayiannakis AJ: Cadherins: an integral role in inflammatory bowel disease and mucosal restitution. J Gastroenterol 2004, 39:413-421

33. Breuhahn K, Baeuerle PA, Peters M, Prang N, Tox U, Kohne-Volland $R$, Dries V, Schirmacher P, Leo E: Expression of epithelial cellular adhesion molecule (Ep-CAM) in chronic (necro-)inflammatory liver diseases and hepatocellular carcinoma. Hepatol Res 2006, 34:50-56

34. Gires O, Kieu C, Fix P, Schmitt B, Munz M, Wollenberg B, Zeidler R: Tumor necrosis factor alpha negatively regulates the expression of the carcinoma-associated antigen epithelial cell adhesion molecule. Cancer 2001, 92:620-628

35. Flieger D, Hoff AS, Sauerbruch T, Schmidt-Wolf IG: Influence of cytokines, monoclonal antibodies and chemotherapeutic drugs on epithelial cell adhesion molecule (EpCAM) and LewisY antigen expression. Clin Exp Immunol 2001, 123:9-14

36. Thurmond LM, Stimmel JB, Ingram AC, Ryan CH, Murray DM, Eberwein DJ, Witherspoon SM, Knick VC: Adenocarcinoma cells exposed in vitro to Navelbine or Taxol increase Ep-CAM expression through a novel mechanism. Cancer Immunol Immunother 2003, 52:429-437

37. Helfrich W, Van Geel M, The TH, de Leij L: Detection of a putative $30-\mathrm{kDa}$ ligand of the cluster-2 antigen. Int J Cancer Suppl 1994, 8:S70-S75 
38. Guillemot JC, Naspetti M, Malergue F, Montcourrier P, Galland F, Naquet P: Ep-CAM transfection in thymic epithelial cell lines triggers the formation of dynamic actin-rich protrusions involved in the organization of epithelial cell layers. Histochem Cell Biol 2001, 116:371-378

39. Würfel J, Rosel M, Seiter S, Claas C, Herlevsen M, Weth R, Zoller M: Metastasis-association of the rat ortholog of the human epithelial glycoprotein antigen EGP314. Oncogene 1999, 18:2323-2334

40. Lipinski M, Parks DR, Rouse RV, Herzenberg LA: Human trophoblast cell-surface antigens defined by monoclonal antibodies. Proc Natl Acad Sci USA 1981, 78:5147-5150

41. Schmidt DS, Klingbeil P, Schnolzer M, Zoller M: CD44 variant isoforms associate with tetraspanins and EpCAM. Exp Cell Res 2004, 297:329-347

42. Moll J, Sleeman J, Kondo K, Hekele A, Plug R, Sherman L, Ponta H, Herrlich P, Schmidt A, Zoller M: Analysis of molecular functions of the tumor metastasis promoting surface molecule CD44v4-v7 using transgenic mice. Princess Takamatsu Symp 1994, 24:142-151

43. Ladwein M, Pape UF, Schmidt DS, Schnolzer M, Fiedler S, Langbein L, Franke WW, Moldenhauer G, Zoller M: The cell-cell adhesion molecule EpCAM interacts directly with the tight junction protein claudin-7. Exp Cell Res 2005, 309:345-357

44. Kominsky SL, Argani P, Korz D, Evron E, Raman V, Garrett E, Rein A, Sauter G, Kallioniemi OP, Sukumar S: Loss of the tight junction protein claudin-7 correlates with histological grade in both ductal carcinoma in situ and invasive ductal carcinoma of the breast. Oncogene 2003, 22:2021-2033

45. Sauer T, Pedersen MK, Ebeltoft K, Naess O: Reduced expression of claudin-7 in fine needle aspirates from breast carcinomas correlate with grading and metastatic disease. Cytopathology 2005, 16:193-198

46. Usami $Y$, Chiba H, Nakayama F, Ueda J, Matsuda $Y$, Sawada N, Komori T, Ito A, Yokozaki H: Reduced expression of claudin-7 correlates with invasion and metastasis in squamous cell carcinoma of the esophagus. Hum Pathol 2006, 37:569-577

47. Claas C, Wahl J, Orlicky DJ, Karaduman H, Schnolzer M, Kempf T, Zoller M: The tetraspanin D6.1A and its molecular partners on rat carcinoma cells. Biochem J 2005, 389:99-110

48. Le Naour F, Andre M, Greco C, Billard M, Sordat B, Emile JF, Lanza $F$, Boucheix C, Rubinstein E: Profiling of the tetraspanin web of human colon cancer cells. Mol Cell Proteomics 2006, 5:845-857

49. Ni J, Hollander D, Sydora B, Panwala C: Adhesion molecule expression and adhesion properties of murine intestinal intraepithelial lymphocyte hybridomas. Cell Immunol 1995, 164:156-160

50. Nochi T, Yuki Y, Terahara K, Hino A, Kunisawa J, Kweon MN, Yamaguchi T, Kiyono H: Biological role of Ep-CAM in the physical interaction between epithelial cells and lymphocytes in intestinal epithelium. Clin Immunol 2004, 113:326-339

51. Osta WA, Chen Y, Mikhitarian K, Mitas M, Salem M, Hannun YA, Cole DJ, Gillanders WE: EpCAM is overexpressed in breast cancer and is a potential target for breast cancer gene therapy. Cancer Res 2004, 64:5818-5824

52. Winter MJ, Nagelkerken B, Mertens AE, Rees-Bakker HA, Briaire-de Bruijn IH, Litvinov SV: Expression of Ep-CAM shifts the state of cadherin-mediated adhesions from strong to weak. Exp Cell Res 2003, 285:50-58

53. Tandon AK, Clark GM, Chamness GC, McGuire WL: Association of the 323/A3 surface glycoprotein with tumor characteristics and behavior in human breast cancer. Cancer Res 1990, 50:3317-3321

54. Jojović M, Adam E, Zangemeister-Wittke U, Schumacher U: Epithelial glycoprotein-2 expression is subject to regulatory processes in epithelial-mesenchymal transitions during metastases: an investigation of human cancers transplanted into severe combined immunodeficient mice. Histochem J 1998, 30:723-729

55. Reiss K, Maretzky T, Ludwig A, Tousseyn T, de Strooper B, Hartmann $\mathrm{D}$, Saftig P: ADAM10 cleavage of $\mathrm{N}$-cadherin and regulation of cellcell adhesion and beta-catenin nuclear signalling. EMBO J 2005, 24:742-752

56. Berthet V, Rigot V, Champion S, Secchi J, Fouchier F, Marvaldi J, Luis $\mathrm{J}$ : Role of endoproteolytic processing in the adhesive and signaling functions of alphavbeta5 integrin. J Biol Chem 2000, 275:33308-33313

57. Okamoto I, Tsuiki H, Kenyon LC, Godwin AK, Emlet DR, HolgadoMadruga M, Lanham IS, Joynes CJ, Vo KT, Guha A, Matsumoto M,
Ushio Y, Saya H, Wong AJ: Proteolytic cleavage of the CD44 adhesion molecule in multiple human tumors. Am J Pathol 2002, 160:441-447

58. Pauli C, Munz M, Kieu C, Mack B, Breinl P, Wollenberg B, Lang S, Zeidler R, Gires O: Tumor-specific glycosylation of the carcinomaassociated epithelial cell adhesion molecule EpCAM in head and neck carcinomas. Cancer Lett 2003, 193:25-32

59. Herrlich P, Morrison H, Sleeman J, Orian-Rousseau V, Konig H, Weg-Remers S, Ponta H: CD44 acts both as a growth- and invasiveness-promoting molecule and as a tumor-suppressing cofactor. Ann NY Acad Sci 2000, 910:106-118

60. Simon B, Podolsky DK, Moldenhauer G, Isselbacher KJ, Gattoni-Celli S, Brand SJ: Epithelial glycoprotein is a member of a family of epithelial cell surface antigens homologous to nidogen, a matrix adhesion protein. Proc Natl Acad Sci USA 1990, 87:2755-2759

61. Münz M, Kieu C, Mack B, Schmitt B, Zeidler R, Gires O: The carcinoma-associated antigen EpCAM upregulates c-myc and induces cell proliferation. Oncogene 2004, 23:5748-5758

62. Münz M, Zeidler R, Gires O: The tumour-associated antigen EpCAM upregulates the fatty acid binding protein E-FABP. Cancer Lett 2005, 225:151-157

63. Furuse M, Hata M, Furuse K, Yoshida Y, Haratake A, Sugitani Y, Noda T, Kubo A, Tsukita S: Claudin-based tight junctions are crucial for the mammalian epidermal barrier: a lesson from claudin-1-deficient mice. J Cell Biol 2002, 156:1099-1111

64. Went PT, Lugli A, Meier S, Bundi M, Mirlacher M, Sauter G, Dirnhofer S: Frequent EpCam protein expression in human carcinomas. Hum Pathol 2004, 35:122-128

65. Szala S, Froehlich M, Scollon M, Kasai Y, Steplewski Z, Koprowski H, Linnenbach AJ: Molecular cloning of cDNA for the carcinoma-associated antigen GA733-2. Proc Natl Acad Sci USA 1990, 87:3542-3546

66. Bumol TF, Marder P, DeHerdt SV, Borowitz MJ, Apelgren LD: Characterization of the human tumor and normal tissue reactivity of the KS1/4 monoclonal antibody. Hybridoma 1988, 7:407-415

67. Moldenhauer G, Momburg F, Moller P, Schwartz R, Hammerling GJ: Epithelium-specific surface glycoprotein of $\mathrm{Mr} 34,000$ is a widely distributed human carcinoma marker. Br J Cancer 1987, 56:714-721

68. Abe H, Kuroki M, Imakiire T, Yamauchi Y, Yamada H, Arakawa F, Kuroki M: Preparation of recombinant MK-1/Ep-CAM and establishment of an ELISA system for determining soluble MK-1/Ep-CAM levels in sera of cancer patients. J Immunol Methods 2002, 270:227-233

69. Houba PH, Boven E, Haisma HJ: Improved characteristics of a human beta-glucuronidase-antibody conjugate after deglycosylation for use in antibody-directed enzyme prodrug therapy. Bioconjug Chem 1996, 7:606-611

70. Sears HF, Herlyn D, Herlyn M, Steplewski Z, Grotzinger P, Koprowski $\mathrm{H}$ : Ex vivo perfusion of human colon with monoclonal anticolorectal cancer antibodies. Cancer 1982, 49:1231-1235

71. Herlyn D, Sears H, lliopoulos D, Lubeck M, Douillard JY, Sindelar W, Tempero M, Mellstedt H, Maher M, Koprowski H: Anti-idiotypic antibodies to monoclonal antibody CO17-1A. Hybridoma 1986, 5(Suppl 1): S51-S58

72. Litvinov SV, Velders MP, Bakker HA, Fleuren GJ, Warnaar SO: EpCAM: a human epithelial antigen is a homophilic cell-cell adhesion molecule. J Cell Biol 1994, 125:437-446

73. Thampoe IJ, Ng JS, Lloyd KO: Biochemical analysis of a human epithelial surface antigen: differential cell expression and processing. Arch Biochem Biophys 1988, 267:342-352

74. De Leij L, Helrich W, Stein R, Mattes MJ: SCLC-cluster-2 antibodies detect the pancarcinoma/epithelial glycoprotein EGP-2. Int J Cancer Suppl 1994, 8:S60-S63

75. Velders MP, Litvinov SV, Warnaar SO, Gorter A, Fleuren GJ, Zurawsk VR Jr, Coney LR: New chimeric anti-pancarcinoma monoclonal antibody with superior cytotoxicity-mediating potency. Cancer Res 1994, 54:1753-1759

76. Strnad J, Hamilton AE, Beavers LS, Gamboa GC, Apelgren LD, Taber LD, Sportsman JR, Bumol TF, Sharp JD, Gadski RA: Molecular cloning and characterization of a human adenocarcinoma/epithelial cell surface antigen complementary DNA. Cancer Res 1989, 49:314-317

77. Ripani E, Sacchetti A, Corda D, Alberti S: Human Trop-2 is a tumorassociated calcium signal transducer. Int J Cancer 1998, 76:671-676 